BNL-NT-08/10

\title{
Equation of state and more from lattice regularized QCD
}

\author{
Frithjof Karsch (for RBC-Bielefeld and hotQCD \\ collaborations) \\ Physics Department, Brookhaven National Laboratory, Upton, NY 11973, USA \\ E-mail: karsch@bnl.gov
}

\begin{abstract}
We present results from a calculation of the QCD equation of state with two light (up, down) and one heavier (strange) quark mass performed on lattices with three different values of the lattice cut-off. We show that also on the finest lattice analyzed by us observables sensitive to deconfinement and chiral symmetry restoration, respectively, vary most rapidly in the same temperature regime.
\end{abstract}

\section{Introduction}

The equation of state (EoS) of strongly interacting elementary particles is one of the most fundamental non-perturbative quantities that numerical studies of lattice regularized QCD will be able to provide as input to the hydrodynamic modeling of the expansion of dense matter formed in heavy ion collisions. At least for vanishing chemical potential, which is appropriate for the conditions met in experiments at RHIC and LHC, lattice calculations of the EoS [1, 2, 3] as well as the transition temperature [4, 5, 6] can now be performed with an almost realistic quark mass spectrum. Calculations at different values of the lattice cut-off allow for a systematic analysis of discretization errors and will soon lead to a controlled extrapolation of the EoS with physical quark masses to the continuum limit.

We present here results from calculation of the EoS on lattices with temporal extent $N_{\tau}=4$ and 6 [3], which correspond to two different sets of the lattice cut-off, $a T \equiv 1 / N_{\tau}$. We furthermore report on preliminary results for the EoS obtained on even finer lattices with temporal extent $N_{\tau}=8$ [7]. We also show results for the temperature dependence of the strange quark number susceptibility and the chiral condensate. These observables are sensitive to different non-perturbative aspects of the QCD transition. Their temperature dependence suggests that the onset of deconfinement as well as the gradual melting of the chiral condensate are correlated and happen in the same temperature regime.

All results presented here are obtained from numerical calculations in lattice regularized (2+1)-flavor QCD using staggered fermions. The calculations have been 

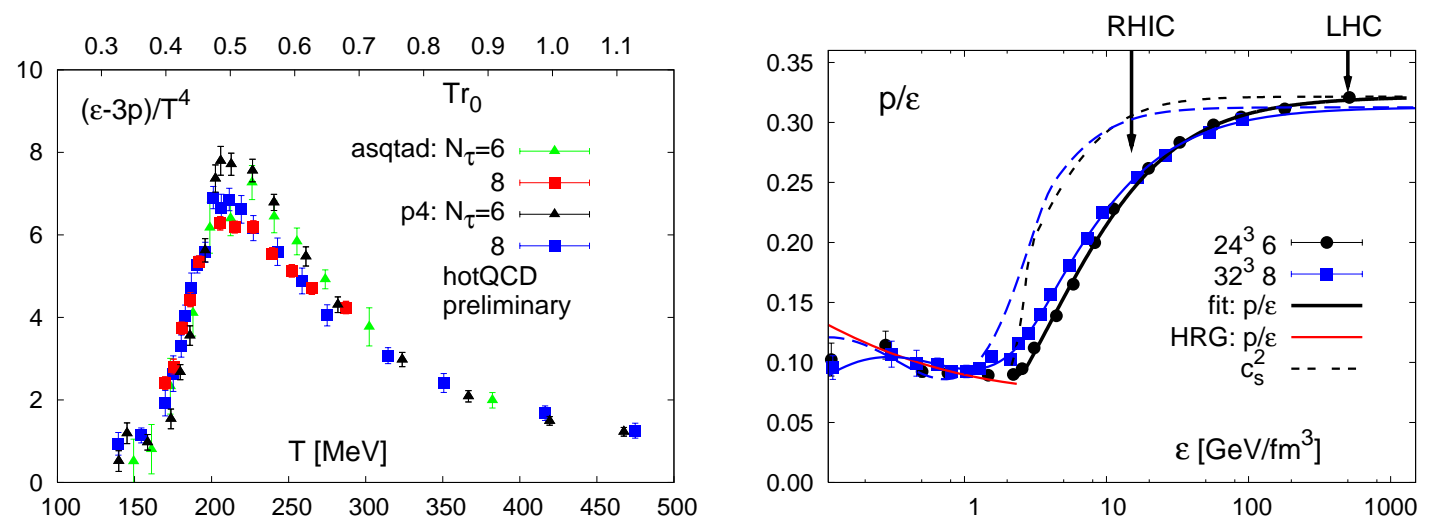

Figure 1. The trace anomaly, $(\epsilon-3 p) / T^{4}$ (left) calculated on lattices with temporal extent $N_{\tau}=6,8$ and the ratio of pressure and energy density as well as the velocity of sound obtained in calculations with the p4fat3 action on $N_{\tau}=6$ (short dashes) [3] and 8 (long dashes) [7. The temperature scale has been obtained from an analysis of the heavy quark potential from which the Sommer scale parameter has been extracted. Its value has been fixed to $r_{0}=0.469 \mathrm{fm}$.

performed with physical values for the strange quark mass and two degenerate light quark masses that correspond to a pion mass of about $220 \mathrm{MeV}$.

\section{The QCD equation of state}

Systematic studies of the QCD EoS are currently performed with two different versions of improved discretization schemes for staggered fermions, the asqtad and p4fat 3 actions. Both actions are constructed such that they remove in the high temperature limit $\mathcal{O}\left(a^{2}\right)$ lattice discretization errors in bulk thermodynamic observables and reduce explicit flavor symmetry breaking effects through the introduction of so-called fat links. In the construction of these actions different strategies have been followed to deal with these lattice artefacts. Both actions have been used for some time to study the QCD equation of state on lattices with temporal extent $N_{\tau}=4$ and 6 [2, 3]. In a joint effort the hotQCD collaboration currently extends the studies of the EoS with these actions to lattices with temporal extent $N_{\tau}=8[8,9$. Like in the earlier calculations performed with the p4fat3 action large spatial lattices are used $\left(N_{\sigma}=4 N_{\tau}\right)$ to get close to the thermodynamic limit.

In Fig. 1 (left) we show results for the trace anomaly, $\Theta^{\mu \mu} \equiv \epsilon-3 p$ in units of $T^{4}$. The figure shows results from calculations performed on lattices with temporal extent $N_{\tau}=6$ and 8 using the asqtad as well as the p4fat 3 actions. It can be seen that both discretization schemes lead to quite good agreement in a wide range of temperatures; although a closer inspection shows still a cut-off dependence of the results. They lead to a reduction of the peak height in $\Theta^{\mu \mu} / T^{4}$, which is located at $T \simeq 200 \mathrm{MeV}$, and lead to a shift of the rapidly rising part of $\Theta^{\mu \mu} / T^{4}$ in the transition region to smaller values of the temperature.

Cut-off effects as well as differences arising from both discretization schemes seem 
to be largest in the vicinity of the maximum of $(\epsilon-3 p) / T^{4}$. This trend has already been observed when comparing results obtained on lattices with temporal extent $N_{\tau}=4$ and 6 [3]. The cut-off dependence observed in $\Theta^{\mu \mu} / T^{4}$ carries over to the calculation of pressure and energy density; the former is obtained by integrating over $\Theta^{\mu \mu} / T^{5}$ and the energy density is then obtained by combining results for $p / T^{4}$ and $(\epsilon-3 p) / T^{4}$. This is apparent in Fig. 1 (right) where we show the ratio $p / \epsilon$ obtained with the p4fat3 action for three different values of the cut-off. Cut-off effects are still visible in the vicinity of the 'softest point' of the EoS, which is related to the peak position of $(\epsilon-3 p) / T^{4}$. In the entire range of energy densities relevant for the expansion of dense matter created at $\mathrm{RHIC}, \epsilon \lesssim 10 \mathrm{GeV} / \mathrm{fm}^{3}$, the ratio $p / \epsilon$ deviates significantly from the conformal, ideal gas value $p / \epsilon=1 / 3$. This also is reflected in the behavior of the velocity of sound, $c_{s}^{2}=\mathrm{d} p / \mathrm{d} \epsilon$, which is shown in Fig. 1 (right) by dashed lines. It starts deviating significantly from the ideal gas value below $\epsilon \simeq 10 \mathrm{GeV} / \mathrm{fm}^{3}$ and reaches a value of about 0.1 in the transition region at energy densities $\epsilon \simeq 1 \mathrm{GeV} / \mathrm{fm}^{3}$.

\section{Deconfinement and chiral symmetry restoration}

The relation between deconfinement and chiral symmetry restoration in QCD has been discussed since a long time. Although both phenomena seem to be related to physics on different length scales lattice calculations seem to suggest that both phenomena happen at approximately the same temperature even at finite, non-zero values of the quark masses when none of the symmetries related to confinement $(Z(3)$ center symmetry) or chiral symmetry breaking $\left(S U_{L}\left(n_{f}\right) \times S U_{R}\left(n_{f}\right)\right)$ are realized exactly. This aspect of the QCD transition received renewed interest recently; as the QCD transition is a crossover rather than a genuine phase transition different observables, that are sensitive to different aspects of the QCD transition, might lead to different transition temperatures. In particular, it has been suggested that observables sensitive to deconfinement may lead to a higher transition temperature than observables sensitive to chiral symmetry restoration 6.

With decreasing quark mass the Polyakov loop looses its role as a genuine order

parameter for deconfinement. It is non-zero at all temperatures. Nonetheless it varies rapidly in the transition region indicating that quark free energies are screened more effectively in the high temperature phase of QCD [3]. Another observable, sensitive to deconfinement is the quark number susceptibility,

$$
\frac{\chi_{q}}{T^{2}}=\frac{1}{V T^{3}} \frac{\partial^{2} \ln Z}{\partial\left(\mu_{q} / T\right)^{2}},
$$

where $q=l, s$ for the light and strange quark sector, respectively. These susceptibilities are sensitive to the degrees of freedom that carry a net number of light or strange quarks, respectively. They too change rapidly in the transition region as the carrier of, e.g. strangeness, are rather heavy strange hadrons at low temperature and single quarks at high temperature. A rapid rise in the susceptibilities thus indicates 'deconfinement'.

In the limit of massless quarks the chiral condensate, $\langle\bar{\psi} \psi\rangle_{q}=T V^{-1} \partial \ln Z / \partial m_{q}$, is 

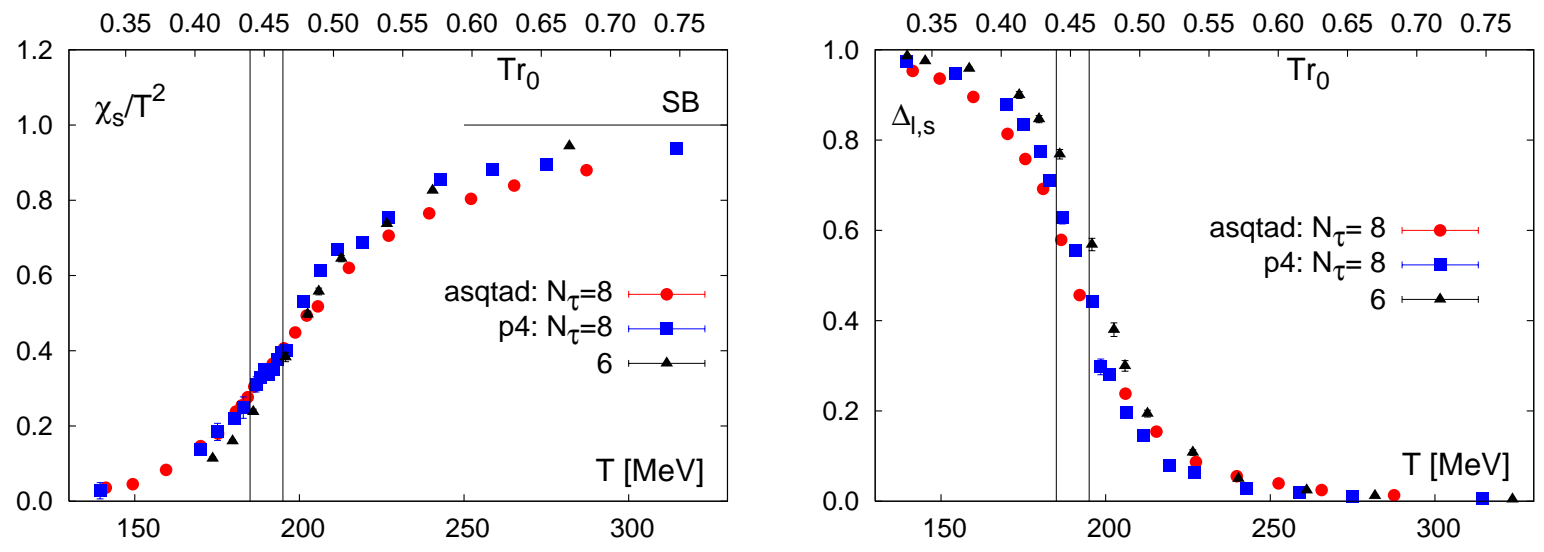

Figure 2. The strange quark number susceptibility (left) and the subtracted chiral condensate normalized to the corresponding zero temperature value (right). The band corresponds to a temperature interval $185 \mathrm{MeV} \leq T \leq 195 \mathrm{MeV}$. Results shown for $N_{\tau}=8$ are preliminary data of the hotQCD collaboration.

an order parameter for chiral symmetry restoration. At non-zero values of the quark mass the condensate receives additive as well as multiplicative renormalization which have to be eliminated to allow for a sensible continuum limit. An appropriate observable is obtain through subtraction of a fraction of the strange quark condensate from the light quark condensate. The difference, taken at finite temperature, can then be normalized with the corresponding zero temperature difference,

$$
\Delta_{l, s}(T)=\frac{\langle\bar{\psi} \psi\rangle_{l, T}-\frac{m_{l}}{m_{s}}\langle\bar{\psi} \psi\rangle_{s, T}}{\langle\bar{\psi} \psi\rangle_{l, 0}-\frac{m_{l}}{m_{s}}\langle\bar{\psi} \psi\rangle_{s, 0}} .
$$

This observable, in addition, has a sensible chiral limit and gives, in this limit, an order parameter that is unity at low temperature and will vanish at $T_{c}$.

In Fig. 2 we show the strange quark number susceptibility (left) as well as $\Delta_{l, s}(T)$ (right). It is apparent from these figures that observables sensitive to deconfinement and chiral symmetry restoration, respectively, vary most rapidly in identical temperature intervals. This interval coincides with the region of most rapid rise in the trace anomaly as well as energy and entropy density.

This manuscript has been authored under contract number DE-AC02-98CH1-886 with the U.S. Department of Energy.

\section{References}

[1] Y. Aoki, Z. Fodor, S. D. Katz and K. K. Szabo, JHEP 0601, 089 (2006).

[2] C. Bernard et al., Phys. Rev. D 75, 094505 (2007).

[3] M. Cheng et al., Phys. Rev. D 77, 014511 (2008).

[4] C. Bernard et al. [MILC Collaboration], Phys. Rev. D 71, 034504 (2005).

[5] M. Cheng et al. (RBC-Bielefeld collaboration), Phys. Rev. D 74, 054507 (2006).

[6] Y. Aoki, Z. Fodor, S. D. Katz and K. K. Szabo, Phys. Lett. B 643, 46 (2006).

[7] HotQCD Collaboration, in preparation.

[8] C. DeTar and R. Gupta [HotQCD Collaboration], PoS (LATTICE 2007) 179.

[9] F. Karsch, PoS (LATTICE 2007) 015. 